\title{
Lymphoscintigraphy and Sentinel Nodes
}

\author{
Valeria M. Moncayo ${ }^{1}$, John N. Aarsvold ${ }^{1,2}$, and Naomi P. Alazraki ${ }^{1,2}$ \\ ${ }^{I}$ Emory University School of Medicine, Atlanta, Georgia; and ${ }^{2}$ VA Medical Center, Atlanta, Georgia
}

Learning Objectives: On successful completion of this activity, participants should be able to describe (1) generic fundamentals of lymphoscintigraphy procedures of sentinel lymph node biopsy (SLNB) protocols; (2) specific injection and imaging components of SLNB protocols used in the management of patients with breast cancer, melanoma, and head and neck malignancies; and (3) specific indications of breast cancer, melanoma, and head and neck malignancies that suggest inclusion of a SLNB in the management of a patient.

Financial Disclosure: The authors of this article have indicated no relevant relationships that could be perceived as a real or apparent conflict of interest.

CME Credit: SNMMI is accredited by the Accreditation Council for Continuing Medical Education (ACCME) to sponsor continuing education for physicians. SNMMI designates each JNM continuing education article for a maximum of 2.0 AMA PRA Category 1 Credits. Physicians should claim only credit commensurate with the extent of their participation in the activity. For CE credit, SAM, and other credit types, participants can access this activity through the SNMMI website (http://www.snmmilearningcenter.org) through June 2018.

It has been validated that sentinel lymph node biopsy (SLNB) shows whether a patient's breast cancer or melanoma has spread to regional lymph nodes. As a result, management of patients with these cancers has been revolutionized. SLNB has replaced axillary lymph node dissection (ALND) as the staging modality of choice for early breast cancer and has replaced complete lymph node dissection as the staging modality of choice for melanoma in patients whose SLNBs indicate no metastases. Recently concluded multicenter, randomized trials for breast cancer with 5- to 10-y outcome data have shown no significant differences in disease-free survival rates or overall survival rates between SLNB and ALND groups but have shown significantly lower morbidity with SLNB than with ALND. The lowest false-negative rates $(5.5 \%-6.7 \%)$ were seen in studies that used preoperative lymphoscintigraphy and dual mapping during surgery. To assess the survival impact of SLNB in melanoma, the Multicenter Selective Lymphadenectomy Trial I was performed. Melanoma-specific survival rates were not different between subjects randomized to SLNB with lymphadenectomy for nodal metastasis on biopsy and subjects randomized to observation with lymphadenectomy for nodal relapse. However, the 10-y disease-free survival rates were better for the SLNB group than for the observation group, specifically among patients with intermediate-thickness melanomas or thick melanomas.

Key Words: lymphoscintigraphy; sentinel lymph node; breast cancer; melanoma

J Nucl Med 2015; 56:901-907

DOI: 10.2967/jnumed.114.141432

$\mathbf{L}$ ymphoscintigraphy refers to planar or tomographic imaging of a radioisotope in lymphatics, usually ${ }^{99 \mathrm{~m}} \mathrm{Tc}$. Identification and localization of all sentinel lymph nodes (SLNs) for surgical biopsy is

Received Mar. 3, 2015; revision accepted Apr. 23, 2015.

For correspondence or reprints contact: Valeria M. Moncayo, Division of Nuclear Medicine and Molecular Imaging, Department of Radiology and Imaging Sciences, Emory University Hospital, 1364 Clifton Rd. NE, 1st Floor, \#D118, Atlanta, GA 30322.

E-mail: vmoncay@emory.edu

Published online Apr. 30, 2015.

COPYRIGHT (C) 2015 by the Society of Nuclear Medicine and Molecular Imaging, Inc. the goal. We discuss SLN procedures that include lymphoscintigraphy in the setting of malignancies to the skin, breast, and head and neck.

\section{LYMPHOSCINTIGRAPHY IN SLN PROCEDURES}

Lymphoscintigraphy for identifying and localizing SLNs has emerged over the past 2 decades as the staging modality of choice for breast cancers and melanomas that are clinically node-negative (1-3). SLNs are regional nodes that directly receive lymph drainage from the primary tumor. If the first lymph node draining a tumor is negative for malignant cells, there is a high probability that the remaining lymph nodes in the relevant primary and subsequent basins will also be negative (4). Removing only SLNs eliminates the need for a full lymphadenectomy, reduces associated adverse effects, and usually results in a better quality of life. There may be several SLNs in a patient, as there may be drainage in multiple directions from the primary lesion. In the case of multiple nodes, the nodes that demonstrate significant radiotracer uptake may all or individually also accumulate metastatic cells; therefore, these should be surgically removed and tested for metastatic cells.

Cabanas, a urologist, introduced SLN biopsy (SLNB) in the management of penile cancer (5). In the 1990s, Morton et al. developed and applied SLNB in the management of patients with melanoma and breast cancer. These protocols used a dye that stains lymph vessels and sentinel nodes blue to make them visible (3). Also in the 1990s, Alex et al. introduced SLNB based on radiotracer techniques. These radiotracer techniques depend on detecting the radiotracer $\gamma$ emissions using a $\gamma$-counting probe or a $\gamma$ camera (2). Lymphoscintigraphy is the dynamic or static imaging of the transit of radiotracer from tumors to SLNs. The imaging often allows a surgeon to easily identify and perform a biopsy of SLNs.

During the years since the initial demonstrations of SLNB protocols, many investigative studies have supported the use of SLNB as a highly reliable method for screening axillary and extraaxillary nodes in early (stages I and II) breast cancer. In the management of early breast cancer, SLNB has replaced the more invasive axillary lymph node dissection (ALND). In melanoma, lymphoscintigraphy provides a means of localizing SLNs to a specific regional drainage area or to multiple regional drainage areas. SLNB has undergone refinement and improvement since the first protocols were reported. SLNB is now widely accepted and used in breast cancer and melanoma ( 6 ) and has been evaluated for potential application to other 
malignancies. We discuss SLNB primarily in the context of breast cancer and melanoma but also remark on its status for head and neck malignancies.

Evidence of the attention to, enthusiasm for, and indeed success of this procedure in aiding management of various cancers is apparent from a Google search for "sentinel+ node" that generated over 500,000 hits and from a PubMed search for "(sentinel) AND node" that generated over 10,000 investigative and educational publications on SLN topics in peer-reviewed journals.

\section{Procedure for SLN Lymphoscintigraphy}

The SLNB procedure requires minimal to no preparation on the part of the patient. Some centers use topical lidocaine and prilocaine cream (EMLA; AstraZeneca) to reduce the pain of needle puncture. Patients who are encouraged to use the cream are instructed on where and how to apply it to unbroken skin. They are asked to do this a minimum of $30 \mathrm{~min}$ before the procedure (7). At the clinic, patients wear a gown after removal of all jewelry and clothing that might negatively affect the lymphoscintigraphy image.

After lymphoscintigraphy, the physician responsible for the injection and imaging procedure communicates directly with the surgeon, and the communications are documented. At the time of surgery, the surgeon has access to the images.

\section{Radiopharmaceuticals and Injection Parameters}

Radiopharmaceuticals used for lymphoscintigraphy include ${ }^{99 \mathrm{~m}}$ Tc-pertechnetate-labeled colloids (particles range from 3 to $5,000 \mathrm{~nm}$ and are often filtered to a narrow size range) and 99mTc-tilmanocept (Lymphoseek; Navidea [mannosyl diethylenetriaminepentaacetic acid (DTPA) dextran]). ${ }^{99 m}$ Tc-tilmanocept was approved by the U.S. Food and Drug Administration for SLN protocols for breast cancer and melanoma in 2013 and for SLN protocols for head and neck squamous cell carcinoma in 2014. Colloids move by lymphatic flow; their migration is dependent on particle size. Most SLN procedures to date have used radiocolloids.

${ }^{99 \mathrm{~m}} \mathrm{Tc}$-tilmanocept is a receptor-based radiotracer. It targets the receptor protein CD-206, found in high concentrations on the surfaces of macrophages and dendritic cells (8). Biochemically, ${ }^{99 m}$ Tc-tilmanocept is a macromolecule comprising multiple units of DTPA and mannose, each covalently attached to a dextran backbone. The mannose acts as a ligand for the receptor, and the DTPA serves as a chelating agent for the ${ }^{99 \mathrm{~m}} \mathrm{Tc}$ labeling. ${ }^{99 \mathrm{~m}} \mathrm{Tc}$-tilmanocept demonstrates faster injection site clearance than ${ }^{99 \mathrm{~m} T c}$-sulfur colloid and equivalent primary SLN uptake (9).

There are no consensus SLN protocols or consensus radiotracer activities or volumes. Practitioners implement and fully test protocols appropriate for their practice environments and patient demographics.

Injection doses range from $3.7 \mathrm{MBq}(3.7 \mathrm{MBq}[0.1 \mathrm{mCi}]$ per intradermal injection) in melanoma to total doses of 185-370 $\mathrm{MBq}$ (185-370 MBq [5-10 $\mathrm{mCi}$ ] per intradermal injection) in breast cancer. For melanoma, injections are most often intradermal. The recommendation is 2-4 intradermal injections of $0.1 \mathrm{~mL}$ each around the tumor site (or around the site of a previously excised tumor) (10). For the extremities, because of rapid lymphatic flow, 2 injections (one medial and one lateral) near the surgical scar or the primary lesion are recommended.

In SLN protocols for breast cancer, injection techniques are more complex and varied. The injection can be either superficial (subdermal, periareolar, or subareolar) or deep (peritumoral) (11-13). Superficial injections are easier to perform than deep injections but may not provide full information on drainage. Specifically, to locate extraaxillary
SLNs, particularly internal mammary SLNs, a protocol should include peritumoral injections (14). Administering peritumoral injections requires more information about the exact location of the tumor than does administering superficial injections. Also, if there has been an excisional biopsy, or if there is a prosthesis, care must be taken not to inject into the dead space of a seroma or into the prosthesis $(10,15)$.

\section{Imaging}

In melanoma, depending on the location of the primary tumor, imaging must cover all possible drainage sites. For example, if the primary tumor is on a patient's trunk, imaging of the left and right axillary and neck regions as well as the left and right pelvis and inguinal regions must be performed (Fig. 1). For extremity tumors, imaging of the axillary and antecubital regions (upper-extremity tumors) or of the inguinal and popliteal regions (lower-extremity tumors) must be performed. For head and neck squamous cell carcinoma, the entire head and neck must be imaged. In particular, all locations distal to the tumor must be imaged, as lymph drainage is generally caudad. Also, for head and neck SLN procedures, practitioners should always keep in mind that many structures in the head and neck are small and vital. Drainage across the body midline is common after injections in the trunk and in the head and neck (Fig. 2) (16). In addition, for some indications SPECT or SPECT/CT may be preferred.

All aspects of the imaging procedure, including skin marking, should be performed with the patient positioned as during surgery.

Dynamic Imaging. In melanoma patients, dynamic imaging begins as soon as the injections are complete. Particularly for head and neck melanomas, immediate imaging reduces the chance of missing a SLN because of rapid drainage from intradermal injections with uptake by superimposed nodes. Imaging at a rate

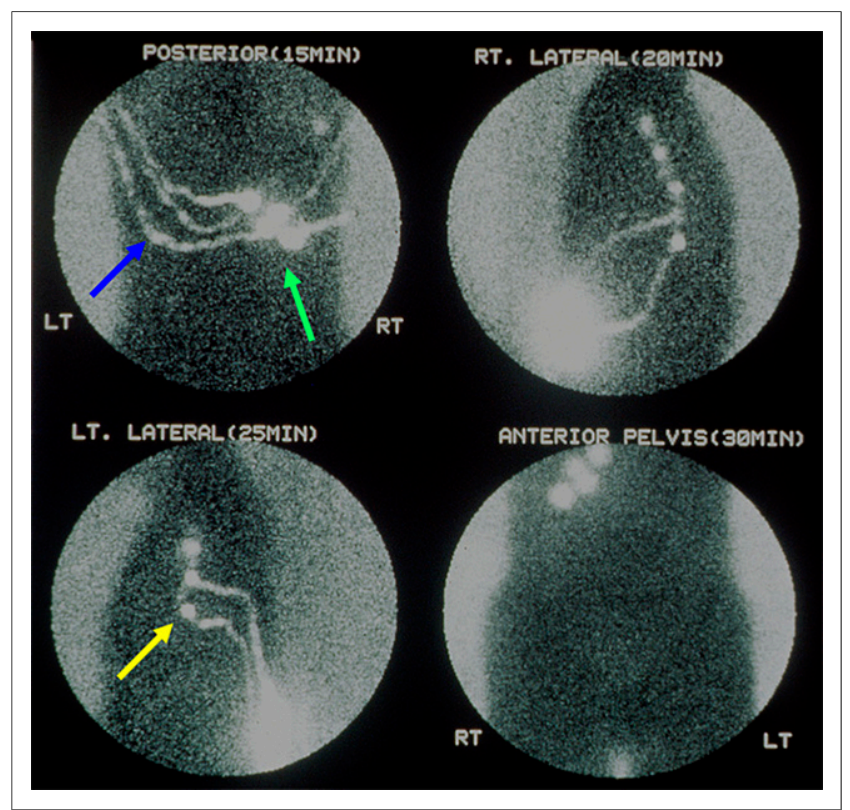

FIGURE 1. Lymphoscintigraphy images of 43-y-old man with melanoma on right mid back. Lymphatic channels are seen arising from tumor site (and injection site) (green arrow) and draining bilaterally to axillary spaces as seen on planar posterior, right lateral, and left lateral views. Visualization of lymphatic channels defines each SLN. A lymphatic channel (blue arrow) terminates on SLN (yellow arrow). Anterior view of pelvis demonstrates no significant activity. No foci of uptake were seen on neck. 


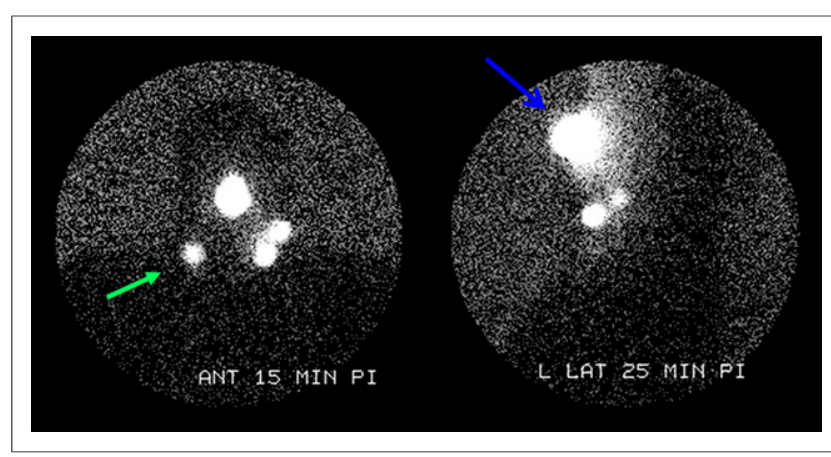

FIGURE 2. Lymphoscintigraphy images of $67-y$-old man with melanoma of nose, $3 \mathrm{~mm}$ in Breslow thickness. Site of injection of $99 \mathrm{mTc}-$ sulfur colloid is visualized on nose on anterior and lateral views (blue arrow). One right (green arrow) and 2 left submandibular lymph nodes were negative for metastasis.

of $1 \mathrm{~s} /$ frame (preferred in the head and neck) or 2 to $5 \mathrm{~s} /$ frame may be performed. Such imaging can visualize the pathways of the radiopharmaceutical and lymph fluid.

Dynamic imaging is not performed routinely in breast cancer protocols because in this context flow is often difficult to image. Also, detection of flow may require long imaging times not generally convenient for practitioners. That said, dynamic images can be acquired in breast cancer protocols and have been proven useful in some cases (Fig. 3).

Planar Imaging. Planar imaging is performed after dynamic imaging or is interspersed with dynamic imaging. Planar imaging is usually performed at 5-min intervals until the SLNs are well visualized. Images in multiple projections are acquired (anterior, lateral oblique, and lateral, as needed, in breast cancer; anterior or posterior and lateral oblique or lateral, if needed, in melanoma). The SLNs in patients with breast cancer or melanoma or head and neck cancer are usually well visualized in 10-60 min; however, visualization can also take several hours.

The use of SPECT or SPECT/CT in SLN protocols has gained importance by virtue of reports documenting that protocols with tomography are more successful than planar imaging in identifying SLNs (17). In melanoma of the head and neck, in which SLNs may be located very close to the primary tumor, the inclusion of SPECT or SPECT/CT may aid in localizing SLNs masked during planar imaging because of their proximity to injection sites (18). In breast cancer, SPECT/CT is an optional technique that is sometimes useful. For example, it can be useful if the SLN is not found, if extraaxillary lymph nodes or an internal mammary lymph node

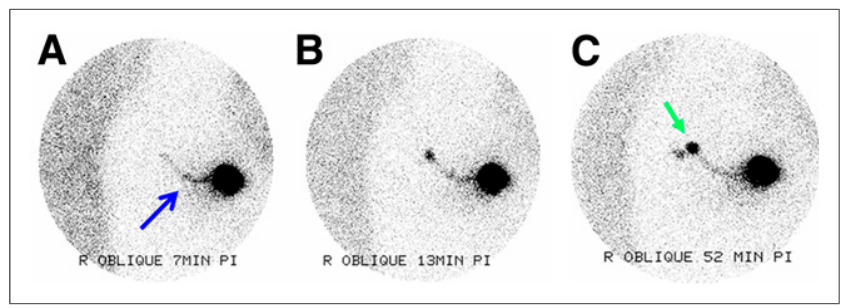

FIGURE 3. Lymphoscintigraphy images of 62-y-old woman with breast cancer. Oblique views demonstrate well-defined lymphatic channel (blue arrow), which was first identified on dynamic images (A); drainage of channel into SLN (B); and SLN (green arrow) and lymphatic channel (C). Secondary node adjacent to SLN was visualized after SLN was visualized.

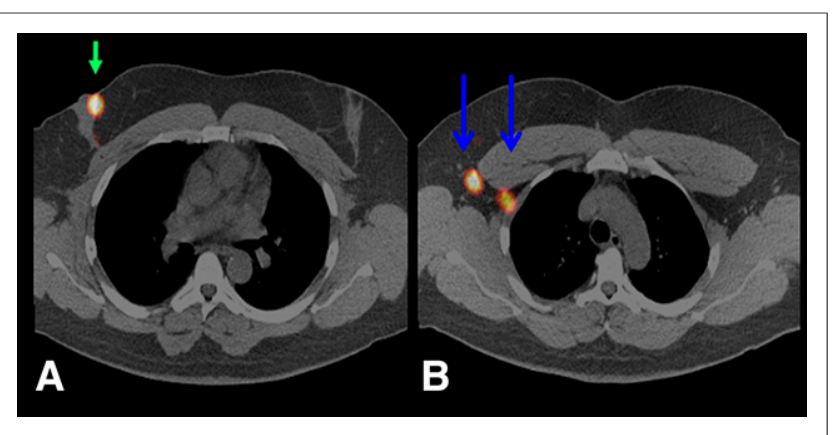

FIGURE 4. SPECT/CT images of 72-y-old man with breast cancer. (A) Radiotracer uptake is seen at peritumoral injection site in right breast (green arrow). (B) The 2 foci of radiotracer uptake correspond to sentinel nodes in right axilla (blue arrows).

is visualized, if contamination is suspected, or if a patient has a high body mass index, previous breast surgery, or contralateral uptake (Fig. 4) (19,20).

Transmission Imaging. In most SLN procedures, planar images are acquired using a flood source $\left({ }^{99 \mathrm{~m}} \mathrm{Tc}\right.$ or $\left.{ }^{57} \mathrm{Co}\right)$. The acquired images include both emission data (from the radiopharmaceutical within the patient) and transmission data (from the flood source). Such images include body contours that provide an anatomic reference for visualized SLNs.

Skin Marking. Once the SLNs are visualized, a $\gamma$-counting probe is used to locate each node and the skin is marked with a pen so that each node can be triangulated and located during surgery. The locations at which to mark the skin can also be determined by superimposing a point source of activity on activity in an SLN while observing the image on a scope. Marking is done in at least 2 projections so that in the operating room the surgeon can predict the depth of the node and triangulate to its position in the body.

When more than one node is visualized in a region, or when more than one is detected at surgery using an intraoperative probe, false-negatives are reduced if the surgeon excises all radioactive nodes. In melanoma studies, in which lymphatic channels are often visualized, locating the SLNs is sometimes straightforward but not always. Surgeons are advised to excise all radioactive nodes when possible.

\section{CANCER INDICATIONS}

\section{Breast Cancer}

In breast cancer, SLNB brings about lower morbidity and mortality rates that are compatible with those of ALND. Additionally, SLNB renders a positive node rate similar to that of ALND.

A single best SLN protocol for use in the management of breast cancer does not exist; several protocols have proven to be successful.

False-Negative Rate and Its Implications. Typically, SLNB aims for a false-negative rate below $5 \%$. The false-negative rate is the proportion of negative sentinel nodes found at biopsy in a patient who actually has positive axillary lymph nodes. The false-negative rate is extremely important because a patient with a false-negative result may have been understaged and possibly undermanaged. A systematic review of 69 studies performed in 2004 as part of guideline development for the American Society of Clinical Oncology found that the false-negative rate of the studies ranged from $0 \%$ to $15 \%$ (21). Importantly, observational studies have reported low rates of axillary recurrence after a negative SLNB result (22). Practitioners should be able to implement a breast 
cancer SLNB protocol that results in identification of at least one SLN in over $95 \%$ of patients whose management includes the specified SLNB protocol (21).

The American Society of Breast Surgeons has published guidelines on credentialing criteria and recommends a minimum of 20 procedures that are proctored or include full axillary dissection before surgeons can perform SLNB on their own (23).

Indications for Lymphoscintigraphy in Breast Cancer. The general indication for use of SLNB in breast cancer is early-stage biopsy-proven breast carcinoma without clinically apparent axillary lymph node metastases. Early stage implies that the cancer has not spread beyond the breast.

There are several established clinical circumstances in which SLNB is used: the primary tumor is T1, T2, or ductal carcinoma in situ (DCIS); the management includes mastectomy; the patient is male; or preoperative systemic therapy is planned (10).

DCIS. DCIS, considered the earliest type of breast cancer, is initially diagnosed using core biopsy. By definition, DCIS does not metastasize to lymph nodes. However, there are several high-risk features, which, if present in the pathologic specimen, suggest more invasive aspects, signaling metastatic potential. The risk for metastatic disease in DCIS is low; however, higher-risk histopathologic features can be missed in up to $40 \%$ of cases that have high-risk features (24). Only $0.1 \%-3 \%$ of DCIS cases have highrisk histopathologic features (24). After surgical resection (lumpectomy or mastectomy) and histopathologic analysis of the specimen, tumors previously diagnosed on core biopsy as DCIS are sometimes reclassified correctly as invasive cancer (25-27). Such reclassification is an upstaging of the patient's diagnosis. If a more invasive cancer is found and there is no clinical evidence of metastasis in the axilla, SLNB is indicated. In DCIS patients for whom lumpectomy or mastectomy is planned, SLNB should be done so that if a more invasive cancer is found, a second operation or a difficult SLNB after the lumpectomy or mastectomy can be avoided (28).

Multicentric Breast Cancer. The use of SLNB in the management of multicentric breast cancer has been and continues to be controversial. Although there is growing evidence that SLNB may become routine in the management of patients with multicentric breast cancer (28), there are questions about how the tracer reaches the SLN and how the procedure is best performed.

According to American Society of Clinical Oncology guidelines, SLN procedures in multicentric breast cancer are acceptable if the primary tumor is smaller than $5 \mathrm{~cm}$. The injections should be superficial and not peritumoral, as in that case the performance of SLNB in multicentric disease is similar to that in unifocal disease (28).

Neoadjuvant Chemotherapy. In locally advanced breast cancer (cancer that includes at least one large tumor), neoadjuvant chemotherapy may be offered for the purpose of shrinking the cancer and thus downstaging it before surgery. This approach is sometimes used even in cases of confirmed axillary metastasis. Such disease has been successfully downstaged in up to $40 \%$ of patients (29).

In early-stage breast cancer, SLNB is performed for staging purposes before neoadjuvant chemotherapy in some clinically nodenegative patients. When this is done, patients receive two surgical interventions: the SLNB before chemotherapy and a lumpectomy or mastectomy after chemotherapy $(15,29)$.

Staging of breast cancer after neoadjuvant chemotherapy can also be an important prognostic factor; therefore, SLNB may be considered after neoadjuvant chemotherapy. In that context, one should recognize that the results represent a new baseline, one different from the baseline before neoadjuvant chemotherapy, even if a pretherapy baseline was not obtained. Multiple metaanalyses suggest that SLN identification can be more than $90 \%$ and false-negative rates less than $12 \%$ in SLNBs after neoadjuvant chemotherapy $(15,30-32)$. Each neoadjuvant chemotherapy patient must be evaluated individually as to whether an SLN procedure is appropriate and, if so, when it should be performed.

The success rate of SLNB after neoadjuvant chemotherapy may be low and the false-negative rate high because of histologic changes in the breast and draining lymphatics due to chemotherapeutic agents. The American College of Surgeons Oncology Group Z0011 (ACOSOG Z0011) Trial was conducted to clarify this controversial issue. The primary endpoint was the false-negative rate of SLNB after chemotherapy in women who presented with N1 disease. The false-negative rate found on ALND was $12.6 \%$, which is considered high (33).

SLN Clinical Trials in Breast Cancer. Several randomized clinical trials investigating SLNB in the management of breast cancer have been conducted over the last 15 y to compare SLNB and ALND. The outcomes of these trials include results on morbidity, mortality, false-negative rate, identification rate, and quality of life.

There are 4 multicenter randomized clinical trials with published 5- to 10-y outcome data. No significant differences have been found in disease-free survival rates $(28,34-38)$ or overall survival rates between SLNB and ALND groups in any of these studies $(28,35-37,39)$.

All the trials showed fewer arm and breast complications and better quality of life in the SLNB groups than in the ALND groups. Fewer doctor visits, less medical care related to morbidity, and increased patient satisfaction are associated with SLNB. Evaluation of arm and breast morbidity included the rates of lymphedema, pain, neuropathy, and reduced range of motion in the ipsilateral arm. At 6-12 mo after surgery, all these outcomes were less frequent in SLNB patients than in ALND patients (34,40-45).

Table 1 summarizes the framework and results of the most important randomized controlled trials comparing SLNB and SLNB + ALND SLNB in the management of breast cancer. Included are the overall survival rate, disease-free survival rate, identification rate, and false-negative rate. The table also notes whether the protocol for each trial included dual mapping or lymphoscintigraphy (34-41).

In the ACOSOG Z0011 Trial, patients with 1 or 2 positive SLNs were randomized intraoperatively to ALND or no ALND. Patients with 3 or more positive lymph nodes were excluded from the study. The main outcome of the study was overall survival. Blue dye alone was used, with no radiotracer.

As seen in Table 1, the lowest false-negative rates were in the Sentinel Node Biopsy Versus Axillary Clearance (SNAC) Trial and the Axillary Lymphatic Mapping Against Nodal Axillary Clearance (ALMANAC) Trial. These trials used a combination of preoperative lymphoscintigraphy and dual mapping during surgery. The Sentinella-GIVOM Trial (Gruppo Interdisciplinare Veneto di Oncologia Mammaria) had the highest false-negative rate. It was conducted in small communities, where the surgeons may not have had significant experience with the procedure before the start of the study. The ALMANAC Trial used a formal training course for all participating surgeons and attempted to standardize the surgical procedures. Trial data suggest that high tumor burdens in SLNs may be a cause of false-negative SLN procedures.

\section{Melanoma}

There is a significant risk of lymph node metastasis in cutaneous invasive melanoma-a risk that increases as Breslow 
TABLE 1

Results of Trials Comparing SLNB and SLNB + ALND in Breast Cancer (34-41)

\begin{tabular}{|c|c|c|c|c|c|c|c|c|c|c|}
\hline \multirow[b]{2}{*}{ Trial } & \multirow[b]{2}{*}{$n$} & \multicolumn{2}{|c|}{ Overall survival } & \multicolumn{3}{|c|}{ Disease-free survival } & \multirow[b]{2}{*}{$\begin{array}{l}\text { Identification } \\
\text { rate }\end{array}$} & \multirow[b]{2}{*}{$\begin{array}{c}\text { False- } \\
\text { negative rate }\end{array}$} & \multirow[b]{2}{*}{ Dual mapping } & \multirow[b]{2}{*}{ Lymphoscintigraphy } \\
\hline & & SLNB & $\begin{array}{l}\text { SLNB + } \\
\text { ALND }\end{array}$ & SLNB & $\begin{array}{l}\text { SLNB + } \\
\text { ALND }\end{array}$ & Years $(n)$ & & & & \\
\hline \multirow[t]{2}{*}{$\begin{array}{l}\text { NSABP B-32 (U.S. \& } \\
\text { Canada) }\end{array}$} & 5,611 & $81.50 \%$ & $82.40 \%$ & $95.00 \%$ & $96.40 \%$ & 5 & $97.20 \%$ & $9.80 \%$ & Yes & No \\
\hline & & & & $91.80 \%$ & $90.30 \%$ & 8 & & & & \\
\hline $\begin{array}{l}\text { Sentinella-GIVOM } \\
\quad \text { (Italy) }\end{array}$ & 697 & $87.60 \%$ & $89.90 \%$ & $94.80 \%$ & $95.50 \%$ & 5 & $95 \%$ & $16.70 \%$ & No & Yes \\
\hline Milan (Italy) & 516 & $89.90 \%$ & $88.80 \%$ & $93.50 \%$ & $89.70 \%$ & 10 & $98 \%$ & $8.80 \%$ & No & Yes \\
\hline $\begin{array}{l}\text { ACOSOG Z0011 } \\
\text { (U.S.) }\end{array}$ & 891 & $83.90 \%$ & $82.20 \%$ & $92.50 \%$ & $91.80 \%$ & 5 & NA & NA & No & No \\
\hline ALMANAC (U.K.) & 1,031 & NA & NA & NA & NA & NA & $96.10 \%$ & $6.70 \%$ & Yes & Yes \\
\hline $\begin{array}{l}\text { SNAC (Australia \& } \\
\text { New Zealand) }\end{array}$ & 1,088 & NA & NA & NA & NA & NA & $95 \%$ & $5.50 \%$ & Yes & Yes \\
\hline
\end{tabular}

thickness increases (Table 2) (46). If all SLNs are negative for metastasis, it is likely there are no metastases; if one or more SLNs are positive, there may be additional metastases.

Staging. Staging of invasive melanoma is based on lesion characteristics such as thickness (Breslow measurement) and level of skin invasion (the Clark level); both are determined by the pathologist from a biopsy sample. The most updated guidelines from the National Comprehensive Cancer Network (47) and the American Joint Committee on Cancer have eliminated the Clark level from staging because it is not an independent prognostic factor when mitotic rate is included in the analysis (48). It is also less predictive of outcome, less reproducible, and more subjective than the Breslow depth (49). Clark levels are still used to predict prognosis in patients with thin $(<1.0 \mathrm{~mm})$ melanomas.

SLNB is recommended in melanomas of clinical stage T1b-T4b without clinically evident locoregional or distant metastasis. SLNB may be offered for lesions of uncertain metastatic potential. According to the most recent guidelines from the American Society of Clinical Oncology, there is insufficient evidence to offer SLNB for thin melanomas; however, if high-risk features are present, such as positive deep margins, ulceration, lymphovascular invasion, age less than $40 \mathrm{y}$, a significant vertical growth phase, or an increased mitotic rate, the procedure is acceptable. If there is a positive SLN, complete lymph node dissection is recommended (50-53).

Clinical Trials in Melanoma. Routine elective complete lymph node dissection reveals metastases in $20 \%$ of patients. Therefore,

TABLE 2

Risks of Lymph Node Metastases for Melanomas of Specified Thicknesses (46)

\begin{tabular}{lc}
\hline Melanoma thickness & Risk \\
\hline Thick $(>4 \mathrm{~mm})$ & $40 \%$ \\
\hline Intermediate $(1-4 \mathrm{~mm})$ & $8 \%-30 \%$ \\
\hline Thin $(<1 \mathrm{~mm})$ & \\
\hline $0.76-1.00 \mathrm{~mm}$ & $5 \%$ \\
$<0.75$ & $1 \%$ \\
\hline
\end{tabular}

approximately $80 \%$ of patients are subjected to surgical morbidity with no clinical benefit. Observation is not a satisfactory approach because it leads to patient anxiety over an uncertain prognosis. The Multicenter Selective Lymphadenectomy Trial I was designed to evaluate whether SLNB with intraoperative lymphatic mapping can detect the $20 \%$ of cases with occult nodal metastasis (54).

The Multicenter Selective Lymphadenectomy Trial I is a phase 3 trial that enrolled 2,001 patients with cutaneous melanomas (predominantly of thin or intermediate thickness). The trial's primary goal was to assess the survival impact of SLNB. Patients were randomly assigned to undergo SLNB with lymphadenectomy for nodal metastasis on biopsy or to be observed with lymphadenectomy for nodal relapse. The study showed that melanoma-specific survival rates were not different between the 2 groups. However, the 10 -y disease-free survival rates were better for the SLNB group than for the observation group among patients with intermediate-thickness melanomas and patients with thick melanomas (54). Many authors do not think this study has conclusively proven the effectiveness of SLNB; however, some interpret the results as indicating that SLNB is the standard of care (55). A 2010 metaanalysis that included nonrandomized studies totalling 2,633 patients reported that SLNB was associated with better survival and suggested that SLNB and complete lymph node dissection might prolong survival in 1 of 5 treated patients after 5 y (56).

The Multicenter Selective Lymphadenectomy Trial II opened in 2005. The investigators plan to enroll 1,925 subjects with sentinel node metastases. If a subject has a positive SLN and meets the study requirements, the subject is randomized to receive either completion lymphadenectomy or observation with nodal ultrasound. Subjects are to be followed for $10 \mathrm{y}$ (57). This trial is designed to answer the question of whether patients with a positive sentinel node need to undergo a completion lymph node dissection. This question stems from the observation that $80 \%$ of patients with a positive sentinel node have no additional disease and do not need complete lymph node dissection (58).

\section{Head and Neck Malignancies}

Head and neck squamous cell cancer is a promising area for SLNB. Oral cavity, oral pharyngeal, and supraglottic squamous cell carcinoma can reveal occult metastasis in $15 \%-60 \%$ of cases 
(59). Multiple studies have validated the procedure, with overall good results $(59,60)$.

Patients with oral squamous cell cancers should undergo SLNB if they present at stage $\mathrm{T} 1$ or $\mathrm{T} 2$ and as clinically neck-negative by palpation, CT, MR imaging, or PET/CT (61). Mapping is performed using preoperative lymphoscintigraphy and a $\gamma$-counting probe (62). Imaging procedures are similar to those used for breast cancer and melanoma, including dynamic and planar imaging and skin marking.

Use of SPECT/CT is optional. Lymph nodes can often be adequately localized with planar imaging alone $(62,63)$. However, the anatomic complexity of the head and neck and the proximity of some primary lesions to SLNs and other important structures suggest SPECT/CT should be included in some cases $(64,65)$.

The radiotracer is injected in small volumes of up to $0.2 \mathrm{~mL}$, containing $0.5 \mathrm{mCi}(20 \mathrm{MBq})$ per injection, in the healthy mucosa surrounding a malignant lesion. Contamination can be avoided if the patient uses a mouthwash or a rinse before swallowing $(61,62)$.

${ }^{99 m}$ Tc-tilmanocept has been approved for SLNB in the management of oral squamous cell carcinomas. Experience with various labeled colloids has been satisfactory, and several are routinely used (61).

Other mapping techniques, such as the use of near-infrared fluorescence tracers (indocyanine green fluorescence navigation, for example), have also been validated in feasibility studies $(66,67)$. The most commonly reported limitation is that the tracer travels rapidly to second-tier nodes.

\section{RADIATION RISKS}

For all the SLN procedures discussed here, the radiation dose to patients is small-well below the limits specified by the International Commission on Radiological Protection. The annual radiation dose to medical professionals (nuclear medicine technologists, nuclear medicine physicians and radiologists, surgeons, surgical support staff, and pathologists) routinely involved in SLN procedures is acceptable - that is, below the occupational annual limits specified by the International Commission on Radiological Protection $(10)$.

If a pregnant woman is to have an SLNB, the absolute need for the procedure should, of course, be confirmed before it is performed. If the procedure is warranted, it should take place; the dose to patient and fetus are small-again within guideline limits (10).

\section{SUMMARY}

SLNB is now the gold standard for lymph node staging in breast cancer and melanoma, offering reduced morbidity and similar mortality rates to those of more invasive lymph node dissections. Consistent use of a chosen technique that includes ${ }^{99 \mathrm{~m} T c-l a b e l e d}$ tracer, blue dye, at least 2 injection sites (superficial and deep), and preoperative imaging is likely to yield a high identification rate and low false-negative rate in breast cancer. In multicenter randomized trials, the lowest false-negative rates $(5.5 \%-6.7 \%)$ were seen in studies that used preoperative lymphoscintigraphy and dual mapping during surgery.

Multicenter, randomized trials for melanoma have shown that specific survival rates were not different between patients randomized to SLNB with lymphadenectomy for nodal metastasis on biopsy and patients randomized to observation with lymphadenectomy for nodal relapse. However, for patients with intermediate-thickness or thick melanomas, the trials have shown a better 10-y disease-free survival rate for the SLNB group than for the observation group.

SLNB protocols for other cancers are applied and are routine in some centers; however, such protocols are not yet widely or routinely used.

\section{REFERENCES}

1. Morton DL, Wanek L, Nizze JA, Elashoff RM, Wong JH. Improved long-term survival after lymphadenectomy of melanoma metastatic to regional nodes: analysis of prognostic factors in 1134 patients from the John Wayne Cancer Clinic. Ann Surg. 1991;214:491-499.

2. Alex JC, Krag DN. Gamma-probe guided localization of lymph nodes. Surg Oncol. 1993;2:137-143.

3. Morton DL, Wen DR, Wong JH, et al. Technical details of intraoperative lymphatic mapping for early stage melanoma. Arch Surg. 1992;127:392-399.

4. Aarsvold JN, Alazraki NP. Update on detection of sentinel lymph nodes in patients with breast cancer. Semin Nucl Med. 2005;35:116-128.

5. Cabanas RM. An approach for the treatment of penile carcinoma. Cancer. 1977;39:456-466.

6. Sondak VK, Wong SL, Gershenwald JE, Thompson JF. Evidence-based clinical practice guidelines on the use of sentinel lymph node biopsy in melanoma. Am Soc Clin Oncol Educ Book. 2013.

7. Krynyckyi BR, Kim CK, Goyenechea MR, Chan PT, Zhang ZY, Machac J. Clinical breast lymphoscintigraphy: optimal techniques for performing studies, image atlas, and analysis of images. Radiographics. 2004;24:121-145.

8. Lymphoseek (technetium Tc $99 \mathrm{~m}$ tilmanocept) [package insert]. Dublin, $\mathrm{OH}$ : Navidea Pharmaceuticals; 2013.

9. Wallace AM, Hoh CK, Vera DR, Darrah DD, Schulteis G. Lymphoseek: a molecular radiopharmaceutical for sentinel node detection. Ann Surg Oncol. 2003;10:531-538.

10. Giammarile F, Alazraki N, Aarsvold JN, et al. The EANM and SNMMI practice guideline for lymphoscintigraphy and sentinel node localization in breast cancer. Eur J Nucl Med Mol Imaging. 2013;40:1932-1947.

11. Johnson CB, Boneti C, Korourian S, Adkins L, Klimberg VS. Intraoperative injection of subareolar or dermal radioisotope results in predictable identification of sentinel lymph nodes in breast cancer. Ann Surg. 2011;254:612-618.

12. Lin KM, Patel TH, Ray A, et al. Intradermal radioisotope is superior to peritumoral blue dye or radioisotope in identifying breast cancer sentinel nodes. $J \mathrm{Am}$ Coll Surg. 2004;199:561-566.

13. Sanli Y, Berberoglu K, Turkmen C, et al. The value of combined peritumoral and subdermal injection techniques for lymphoscintigraphy in detection of sentinel lymph node in breast cancer. Clin Nucl Med. 2006;31:690-693.

14. Rodier JF, Velten M, Wilt M, et al. Prospective multicentric randomized study comparing periareolar and peritumoral injection of radiotracer and blue dye for the detection of sentinel lymph node in breast sparing procedures: FRANSENODE trial. J Clin Oncol. 2007;25:3664-3669.

15. Moncayo VM, Aarsvold JN, Grant SF, Bartley SC, Alazraki NP. Status of sentinel lymph node for breast cancer. Semin Nucl Med. 2013;43:281-293.

16. Uren RF, Howman-Giles R, Thompson JF. Patterns of lymphatic drainage from the skin in patients with melanoma. J Nucl Med. 2003;44:570-582.

17. van der Ploeg IM, Valdes Olmos RA, Nieweg OE, Rutgers EJ, Kroon BB, Hoefnagel CA. The additional value of SPECT/CT in lymphatic mapping in breast cancer and melanoma. J Nucl Med. 2007;48:1756-1760.

18. Mar MV, Miller SA, Kim EE, Macapinlac HA. Evaluation and localization of lymphatic drainage and sentinel lymph nodes in patients with head and neck melanomas by hybrid SPECT/CT lymphoscintigraphic imaging. J Nucl Med Technol. 2007;35:10-16.

19. Uren RF, Howman-Giles R, Chung DK, et al. SPECT/CT scans allow precise anatomical location of sentinel lymph nodes in breast cancer and redefine lymphatic drainage from the breast to the axilla. Breast. 2012;21:480-486.

20. van der Ploeg IM, Valdes Olmos R, Kroon B, Nieweg O. The hybrid SPECT/CT as an additional lymphatic mapping tool in patients with breast cancer. World $J$ Surg. 2008;32:1930-1934.

21. Lyman GH, Giuliano AE, Somerfield MR, et al. American Society of Clinical Oncology guideline recommendations for sentinel lymph node biopsy in earlystage breast cancer. J Clin Oncol. 2005;23:7703-7720.

22. van der Ploeg IM, Nieweg OE, van Rijk MC, Valdes Olmos RA, Kroon BB. Axillary recurrence after a tumour-negative sentinel node biopsy in breast cancer patients: a systematic review and meta-analysis of the literature. Eur J Surg Oncol. 2008;34:1277-1284.

23. Tafra L, McMasters KM, Whitworth P, Edwards MJ. Credentialing issues with sentinel lymph node staging for breast cancer. Am J Surg. 2000;180:268-273. 
24. Intra M, Rotmensz N, Veronesi P, et al. Sentinel node biopsy is not a standard procedure in ductal carcinoma in situ of the breast: the experience of the European Institute of Oncology on 854 patients in 10 years. Ann Surg. 2008;247:315-319.

25. Camp R, Feezor R, Kasraeian A, et al. Sentinel lymph node biopsy for ductal carcinoma in situ: an evolving approach at the University of Florida. Breast J. 2005;11:394-397.

26. Pechlivanides G, Vassilaros D, Tsimpanis A, Apostolopoulou A, Vasilaros S. Sentinel node biopsy for breast cancer patients: issues for discussion and our practice. Patholog Res Int. 2011:109712.

27. Ansari B, Boughey JC. Sentinel lymph node surgery in uncommon clinical circumstances. Surg Oncol Clin N Am. 2010;19:539-553.

28. Hindié E, Groheux D, Brenot-Rossi I, Rubello D, Moretti JL, Espie M. The sentinel node procedure in breast cancer: nuclear medicine as the starting point. J Nucl Med. 2011;52:405-414.

29. Kang E, Chung IY, Han SA, et al. Feasibility of sentinel lymph node biopsy in breast cancer patients with initial axillary lymph node metastasis after primary systemic therapy. J. Breast Cancer. 2011;14:147-152.

30. Kelly AM, Dwamena B, Cronin P, Carlos RC. Breast cancer sentinel node identification and classification after neoadjuvant chemotherapy-systematic review and meta analysis. Acad Radiol. 2009;16:551-563.

31. van Deurzen CH, Vriens BE, Tjan-Heijnen VC, et al. Accuracy of sentinel node biopsy after neoadjuvant chemotherapy in breast cancer patients: a systematic review. Eur J Cancer. 2009;45:3124-3130.

32. Xing Y, Foy M, Cox D, Kuerer H, Hunt K, Cormier J. Meta-analysis of sentinel lymph node biopsy after preoperative chemotherapy in patients with breast cancer. Br J Surg. 2006;93:539-546.

33. Boughey JC, Suman VJ, Mittendorf EA, et al. Sentinel lymph node surgery after neoadjuvant chemotherapy in patients with node-positive breast cancer: the ACOSOG Z1071 (Alliance) clinical trial. JAMA. 2013;310:1455-1461.

34. Ashikaga T, Krag DN, Land SR, et al. Morbidity results from the NSABP B-32 trial comparing sentinel lymph node dissection versus axillary dissection. J Surg Oncol. 2010;102:111-118.

35. Giuliano AE, McCall L, Beitsch P, et al. Locoregional recurrence after sentinel lymph node dissection with or without axillary dissection in patients with sentinel lymph node metastases: the American College of Surgeons Oncology Group Z0011 randomized trial. Ann Surg. 2010;252:426-432.

36. Krag DN, Anderson SJ, Julian TB, et al. Sentinel-lymph-node resection compared with conventional axillary-lymph-node dissection in clinically nodenegative patients with breast cancer: overall survival findings from the NSABP B-32 randomised phase 3 trial. Lancet Oncol. 2010;11:927-933.

37. Veronesi U, Viale G, Paganelli G, et al. Sentinel lymph node biopsy in breast cancer: ten-year results of a randomized controlled study. Ann Surg. 2010;251:595600 .

38. Zavagno G, De Salvo GL, Scalco G, et al. A randomized clinical trial on sentinel lymph node biopsy versus axillary lymph node dissection in breast cancer: results of the Sentinella/GIVOM trial. Ann Surg. 2008;247:207-213.

39. Gentilini O, Veronesi P, Botteri E, et al. Sentinel lymph node biopsy in multicentric breast cancer: five-year results in a large series from a single institution. Ann Surg Oncol. 2011;18:2879-2884.

40. Del Bianco P, Zavagno G, Burelli P, et al. Morbidity comparison of sentinel lymph node biopsy versus conventional axillary lymph node dissection for breast cancer patients: results of the sentinella-GIVOM Italian randomised clinical trial. Eur J Surg Oncol. 2008;34:508-513.

41. Fleissig A, Fallowfield LJ, Langridge CI, et al. Post-operative arm morbidity and quality of life: results of the ALMANAC randomised trial comparing sentinel node biopsy with standard axillary treatment in the management of patients with early breast cancer. Breast Cancer Res Treat. 2006;95:279-293.

42. Fougo JL, Dinis-Ribeiro M, Araujo C, et al. Impact of lymphadenectomy on axillary recurrence and morbidity of the upper limb in breast cancer patients with negative sentinel node: a prospective randomised study. Cir Esp. 2011;89: 307-316.

43. Goyal A, Newcombe RG, Chhabra A, Mansel RE. Morbidity in breast cancer patients with sentinel node metastases undergoing delayed axillary lymph node dissection (ALND) compared with immediate ALND. Ann Surg Oncol. 2008;15:262267.

44. Helms G, Kuhn T, Moser L, Remmel E, Kreienberg R. Shoulder-arm morbidity in patients with sentinel node biopsy and complete axillary dissection: data from a prospective randomised trial. Eur J Surg Oncol. 2009;35:696-701.
45. Purushotham AD, Upponi S, Klevesath MB, et al. Morbidity after sentinel lymph node biopsy in primary breast cancer: results from a randomized controlled trial. $J$ Clin Oncol. 2005;23:4312-4321.

46. Chakera AH, Hesse B, Burak Z, et al. EANM-EORTC general recommendations for sentinel node diagnostics in melanoma. Eur J Nucl Med Mol Imaging. 2009;36:1713-1742.

47. NCCN guidelines for treatment of cancer by site: penile cancer. National Comprehensive Cancer Center website. http://www.nccn.org/professionals/physician_gls/ f_guidelines.asp\#site. Accessed April 27, 2015.

48. Balch CM, Gershenwald JE, Soong SJ, et al. Final version of 2009 AJCC melanoma staging and classification. J Clin Oncol. 2009;27:6199-6206.

49. McMasters KM, Wong SL, Edwards MJ, et al. Factors that predict the presence of sentinel lymph node metastasis in patients with melanoma. Surgery. 2001;130:151-156.

50. Wong SL, Balch CM, Hurley P, et al. Sentinel lymph node biopsy for melanoma: American Society of Clinical Oncology and Society of Surgical Oncology joint clinical practice guideline. J Clin Oncol. 2012;30:2912-2918.

51. Karakousis GC, Gimotty PA, Czerniecki BJ, et al. Regional nodal metastatic disease is the strongest predictor of survival in patients with thin vertical growth phase melanomas: a case for SLN staging biopsy in these patients. Ann Surg Oncol. 2007;14:1596-1603.

52. Karakousis GC, Gimotty PA, Botbyl JD, et al. Predictors of regional nodal disease in patients with thin melanomas. Ann Surg Oncol. 2006;13:533-541.

53. Bedrosian I, Faries MB, Guerry DT, et al. Incidence of sentinel node metastasis in patients with thin primary melanoma $(<$ or $=1 \mathrm{~mm})$ with vertical growth phase. Ann Surg Oncol. 2000;7:262-267.

54. Morton DL, Thompson JF, Cochran AJ, et al. Final trial report of sentinel-node biopsy versus nodal observation in melanoma. N Engl J Med. 2014;370:599-609.

55. Pasquali S, Spillane A. Contemporary controversies and perspectives in the staging and treatment of patients with lymph node metastasis from melanoma, especially with regards positive sentinel lymph node biopsy. Cancer Treat Rev. 2014;40:893-899.

56. Pasquali S, Mocellin S, Campana LG, et al. Early (sentinel lymph node biopsyguided) versus delayed lymphadenectomy in melanoma patients with lymph node metastases: personal experience and literature meta-analysis. Cancer. 2010;116:12011209.

57. Morton DL. Overview and update of the phase III Multicenter Selective Lymphadenectomy Trials (MSLT-I and MSLT-II) in melanoma. Clin Exp Metastasis. 2012;29:699-706.

58. McNeil C. Does every melanoma patient with a positive sentinel node need more lymph nodes removed? The ASCO Post website. http://www.ascopost.com/ issues/may-1,-2013/higher-her2-expression-linked-to-best-results-with-t-dm1-inpatients-with.aspx. Published May 1, 2013. Accessed April 27, 2015.

59. Alex JC, Sasaki CT, Krag DN, Wenig B, Pyle PB. Sentinel lymph node radiolocalization in head and neck squamous cell carcinoma. Laryngoscope. 2000; 110:198-203.

60. Radkani P, Mesko TW, Paramo JC. Validation of the sentinel lymph node biopsy technique in head and neck cancers of the oral cavity. Am Surg. 2013;79:1295-1297.

61. Alkureishi LW, Burak Z, Alvarez JA, et al. Joint practice guidelines for radionuclide lymphoscintigraphy for sentinel node localization in oral/oropharyngeal squamous cell carcinoma. Eur J Nucl Med Mol Imaging. 2009;36:1915-1936.

62. Stoeckli SJ, Steinert H, Pfaltz M, Schmid S. Sentinel lymph node evaluation in squamous cell carcinoma of the head and neck. Otolaryngol Head Neck Surg. 2001; 125:221-226.

63. Ross GL, Soutar DS, Gordon MacDonald D, et al. Sentinel node biopsy in head and neck cancer: preliminary results of a multicenter trial. Ann Surg Oncol. 2004;11:690-696.

64. Bilde A, Von Buchwald C, Mortensen J, et al. The role of SPECT-CT in the lymphoscintigraphic identification of sentinel nodes in patients with oral cancer. Acta Otolaryngol. 2006;126:1096-1103.

65. Wagner A, Schicho K, Glaser C, et al. SPECT-CT for topographic mapping of sentinel lymph nodes prior to gamma probe-guided biopsy in head and neck squamous cell carcinoma. J Craniomaxillofac Surg. 2004;32:343-349.

66. Schaafsma BE, Mieog JS, Hutteman M, et al. The clinical use of indocyanine green as a near-infrared fluorescent contrast agent for image-guided oncologic surgery. J Surg Oncol. 2011;104:323-332.

67. van der Vorst JR, Schaafsma BE, Verbeek FP, et al. Near-infrared fluorescence sentinel lymph node mapping of the oral cavity in head and neck cancer patients. Oral Oncol. 2013;49:15-19. 